J. Gynäkol. Endokrinol. 2018 ·28:107-109 https://doi.org/10.1007/s41974-018-0060-x

C Der/die Autor(en) 2018

CrossMark

\section{Christoph Brezinka}

Universitätsklinik für gynäkologische Endokrinologie und Reproduktionsmedizin, Medizinische Universität Innsbruck, Innsbruck, Österreich

\title{
Der verwirrende Nebenbefund im gynäkologischen Ultraschall
}

\begin{abstract}
Immer wieder kommt es vor, dass man im gynäkologischen Ultraschall plötzlich Strukturen sieht, bei denen man nicht weiß, welchem Organ man sie zuordnen soll, und bei denen den Untersucher in der ersten Sekunde zunächst das Gefühl beschleicht, dass die Strukturen da nichts verloren haben. Am Beispiel von Implantaten, Zysten und Nierendystopien soll die abwechslungsreiche Welt der verwirrenden Nebenbefunde aufgezeigt und erläutert werden.
\end{abstract}

\section{Reservoire von Kathetern und künstlichen Sphinkteren}

Spätestens beim Einbringen der Vaginalsonde in die Scheide sollte bei der Untersuchung ein liegender Blasenkatheter aufgefallen sein, und das von ihm hervorgerufene Bild einer kreisrunden Struktur in dem Bereich vor dem Uterus sollte nicht allzu viel Staunen auslösen (• Abb. 1). Wenn etwas Harn in der Blase ist, ist das Bild sofort plausibel (• Abb. 2), wenn nur der Katheter in der Blase liegt, kann dies zunächst verwirren, wenn man nicht daran denkt.

Artifizielle Sphinktere für Harn- oder Stuhlinkontinenz brauchen Depots, wobei ein mit physiologischer Kochsalzlösung gefülltes Ballonreservoir auch intraabdominal in unmittelbarer Nähe der Ovarien platziert werden kann (• Abb. 3). Betroffene Patientinnen wissen darüber immer Bescheid, wenn man in den Unterlagen nichts findet, sollte man sie danach fragen. Vor allem sollte ein voreiliges Anpunktieren dieser Ballonreservoire - sei es aus diagnostischen Gründen, sei es, weil man es für einen Follikel hält - unterbleiben. Rücksprache mit Vertretern des Fachs, die üblicherweise solche Implantate legen, kann sehr aufschlussreich sein und Schaden für die Patientin verhindern.

\section{Beckenniere}

Beckennieren sind bei Frauen wesentlich häufiger als bei Männern und häufiger auf der linken als auf der rechten Seite. Meist sind sie den Patientinnen bekannt, sie können aber durchaus auch bei einem Vaginalultraschall erstmals auffallen. Da die Rotation der Nieren im Zuge der embryonalen Entwicklung einer Nierendystopie auch gestört ist, liegt das Nierenbecken ventral des Nierenparenchyms und nicht medial. Die Beckenniere sieht also nicht aus wie eine „typische“ Niere im Ultraschall und kann auf den ersten Blick auch für einen soliden Ovarialtumor gehalten werden (-Abb. 4). Steht die Diagnose fest, sollte die Gelegenheit genutzt werden, gleich mit 3-D-Ultraschall nach
Uterusfehlbildungen zu fahnden, da diese bei Nierendystopien gehäuft auftreten [1].

\section{Die Gartner-Gang-Zyste}

Im indifferenten Stadium haben alle Embryonen 2 Geschlechtsgänge: die Müllerschen Gänge (für Entwicklung zur Frau) und die Wolffschen Gänge (für Entwicklung zum Mann). Aus den Müllerschen Gängen entstehen die Tube, das Corpus und die Cervix uteri sowie der obere Anteil der Vagina. Die Wolffschen Gänge obliterieren, weil der stimulierende Faktor aus den Hoden fehlt. Selten bleiben Residuen der Wolffschen Gänge zurück, die sich als Gartner-Gang-Zysten in der Vaginalwand präsentieren können, oder - noch seltener - als Zysten im Mesovar oder dem Ligamentum latum [2]. Die Morgagni-Hydatiden, die man häufig im Bereich des Fimbrientrichters sieht, sind

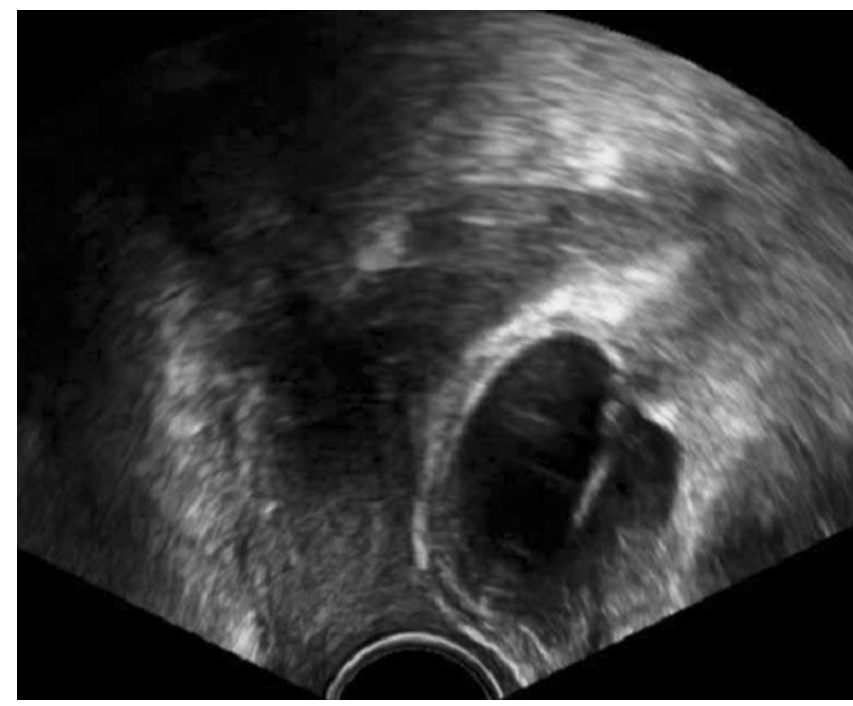

Abb. $1 \triangleleft$ Kreisrunde, homogen echodens demarkierte, echoarme Struktur mit zentralem echodensem Streifen anterior des Uterus gelegen ... was ist das? 


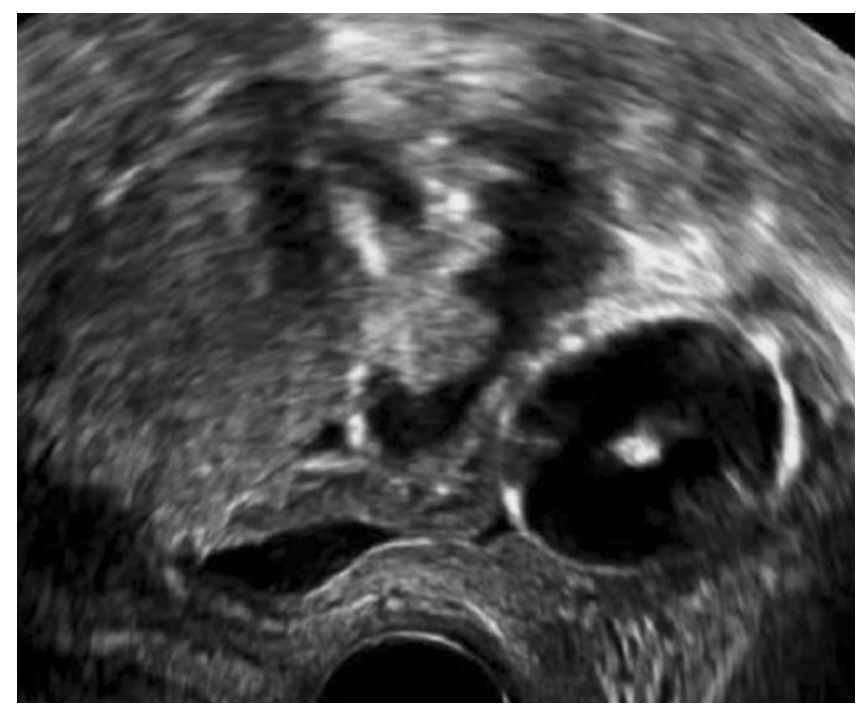

Abb. 2 \Sobald die Blase nur etwas gefüllt ist, löst sich das Rätsel von - Abb. 1 und es zeigt sich der Ballon eines liegenden Blasenverweilkatheters

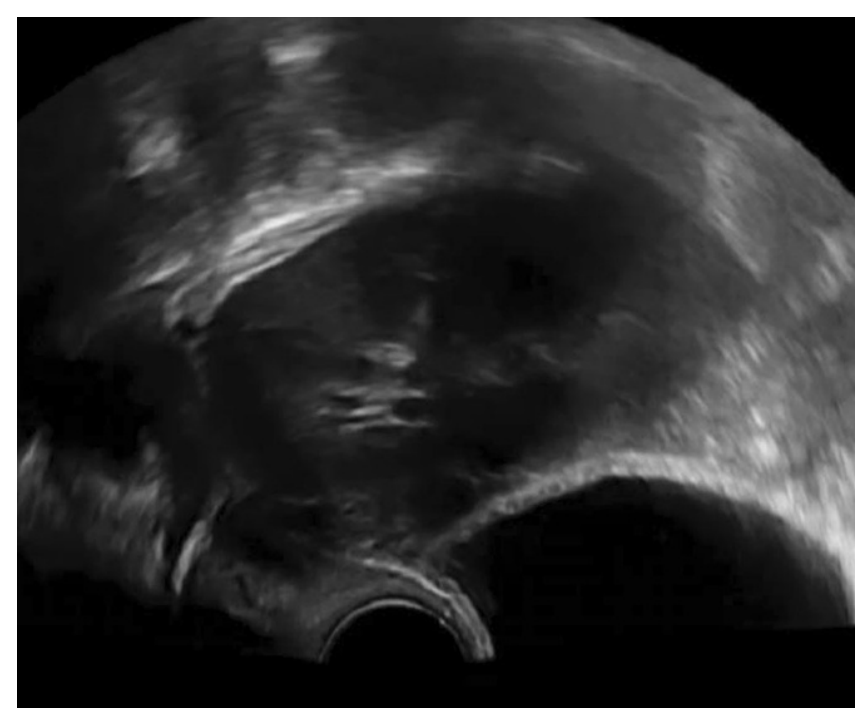

Abb. 4 ム Hier liegt die Beckenniere zwischen Zervix (unten) und Harnblase (rechts im Bild)

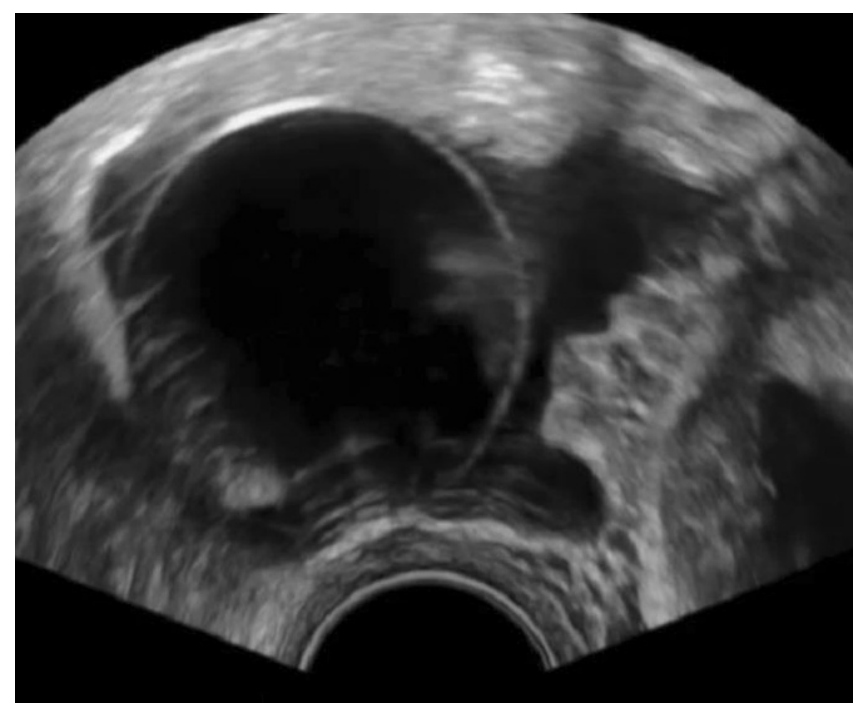

Abb. $3 \Delta$ Das Reservoir eines Scott-Sphinkters (American Medical Systems, Minnetonka, MI,USA) kann unmittelbarneben den Ovarien liegen und sollte im Zuge reproduktionsmedizinischer Maßnahmen möglichst nicht anpunktiert werden

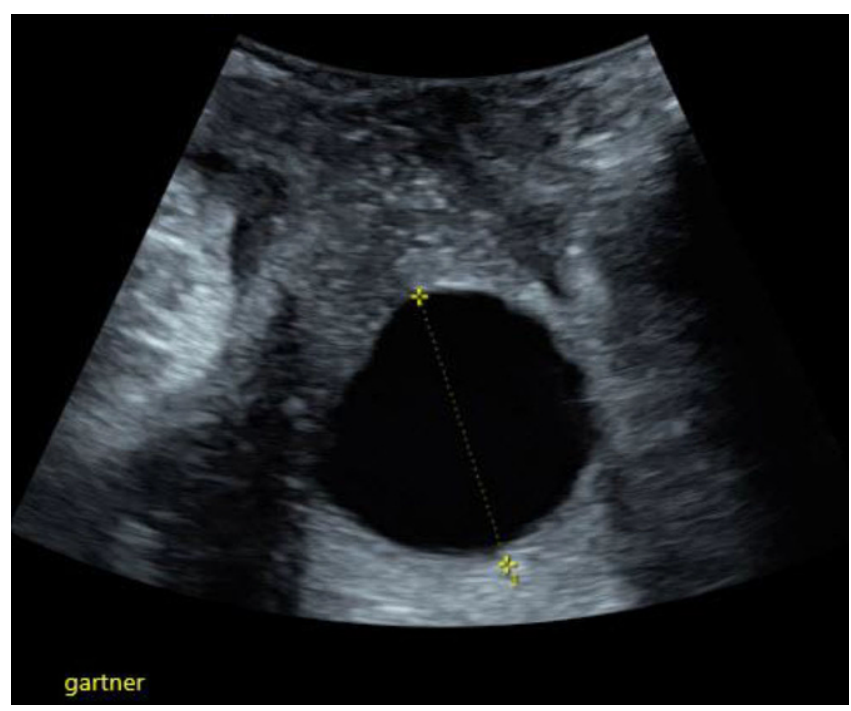

Abb. 5 ム Mit dem Abdominalschallkopf von perineal geschallte, seit 4 Jahren konstante Gartner-Gang-Zyste im Bereich des Fornix vaginae dagegen der anatomisch oberste Rest des Müllerschen Gangs.

Der Anatom und Begründer der Histologie Marcello Malpighi (1628-1694) hat in seinem umfangreichen Werk auch einmal eine Zyste in der Vaginalwand beschrieben. Der dänische Arzt Hermann Treschow Gartner (1785-1827) war aber der erste, der diese Zysten bei der Frau von den Resten des Mesonephrons (Wolffscher Gang) herleiten konnte. Bis heute heißen sie auf Französisch und Italienisch „canal de Malpighi-Gartner“, in allen an- deren Sprachen „Gartner's duct“ oder, wie im Deutschen, Gartner-Gang-Zysten [3].

Gartner-Gang-Zysten sind im Prinzip harmlos, das Innere der Zysten ist meist dünnflüssig, kann aber auch gallertartige Konsistenz haben [4]. Selten - und dann eher im jugendlichen Alter - können sie sehr groß werden [5]. Es gibt einzelne Fallberichte von malignen Prozessen im Vaginalbereich, die aus Gartner-GangZysten entstanden sein sollen [6, 7]. Beobachten mittels Bildgebung ist bei symptomlosen Patientinnen einer Punktion oder Exzision auf jeden Fall vorzuziehen (- Abb. 5 und 6).

\section{NuvaRing}

Zum Abschluss sollte noch der NuvaRing erwähnt werden, der ein charakteristisches sonographisches Erscheinungsbild aufweist - im normalen Schnittbild hat er eine nahezu rechteckige Form, hinter der ein Schatten liegt, wie man ihn von Hormonspiralen kennt (• Abb. 7). Es gibt Fallberichte von Anwenderinnen, die den 


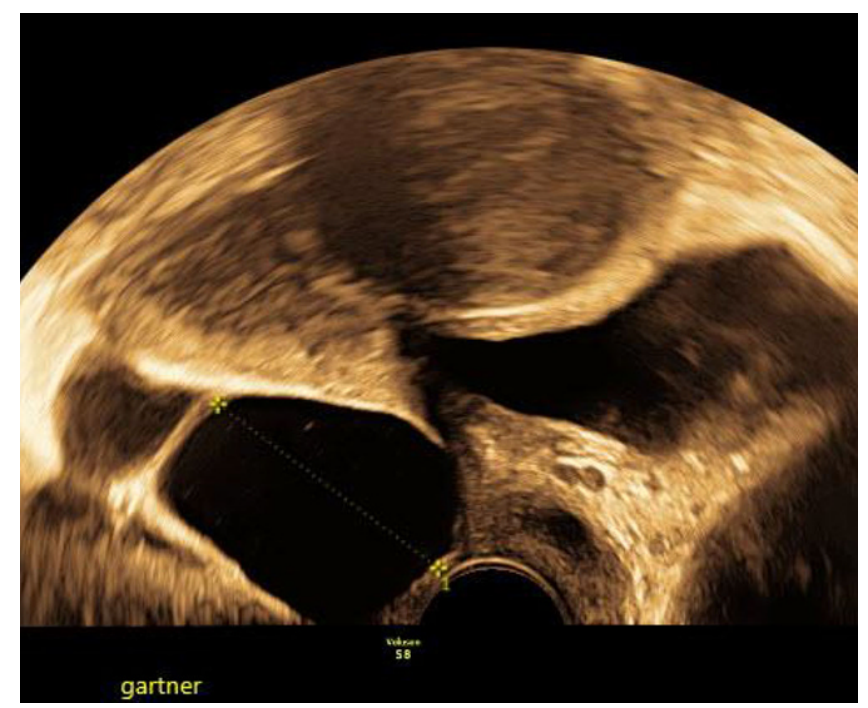

Abb. 6 × Über Jahre konstante Gartner-Gang-Zyste, die trotz der unmittelbaren Nähe zur Blase der Patientin nie Beschwerden machte

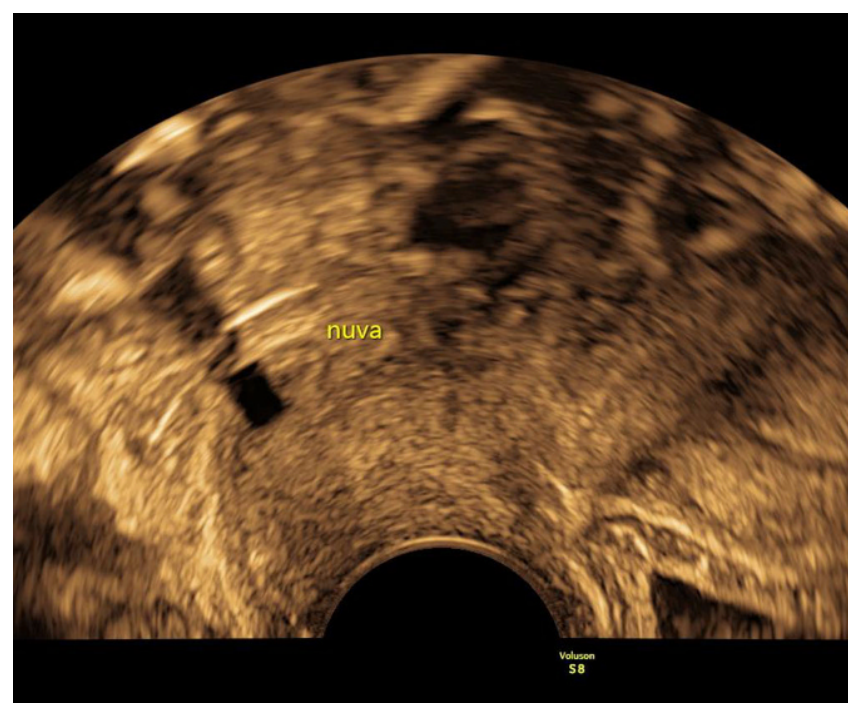

Abb. 7 A Ein NuvaRing imponiert als rechteckiger, echoarmer Block unmittelbar hinter der Zervix
NuvaRing versehentlich in der Harnblase platzierten, dieser kann mit entsprechend gefüllter Blase im Ultraschall gesehen und zystoskopisch entfernt werden [8].

Ultraschall wird in allen klinischen Fächern immer mehr zu einer Point-ofcare-Technik, wobei in jedem Untersuchungsraum ein Ultraschallgerät steht und keine Untersuchung ohne Ultraschallbefund vollständig ist. Dies bringt mit sich, dass viel diagnostisches Wissen autodidaktisch erworben werden muss, was nur bei besonderer Aufmerksamkeit auf Haupt- und Nebenbefunde gelingen kann. Die Wahl der richtigen „presets“, Dokumentation und Archivierung gehören ebenso dazu wie das nie ablassende Interesse daran, ob sich die eigene Diagnose als richtig herausgestellt hat.

\section{Korrespondenzadresse}

\section{Ao. Univ.-Prof. Dr. C. Brezinka}

Universitätsklinik für gynäkologische

Endokrinologie und Reproduktionsmedizin,

Medizinische Universität Innsbruck

Anichstraße 35, 6020 Innsbruck, Österreich

christoph.brezinka@i-med.ac.at

Funding. Open access funding provided by University of Innsbruck and Medical University of Innsbruck.

\section{Einhaltung ethischer Richtlinien}

Interessenkonflikt. C. Brezinka gibt an, dass kein Interessenkonflikt besteht.

Dieser Beitrag beinhaltet keine vom Autor durchgeführten Studien an Menschen oder Tieren.

Open Access. Dieser Artikel wird unter der Creative Commons Namensnennung 4.0 International Lizenz (http://creativecommons.org/licenses/by/4.0/deed. de) veröffentlicht, welche die Nutzung, Vervielfältigung, Bearbeitung, Verbreitung und Wiedergabe in jeglichem Medium und Format erlaubt, sofern Sie den/die ursprünglichen Autor(en) und die Quelle ordnungsgemäß nennen, einen Linkzur Creative Commons Lizenz beifügen und angeben, ob Änderungen vorgenommen wurden.

\section{Literatur}

1. Kapczuk K, Friebe Z, Iwaniec K, Kedzia W (2018) Obstructive Mullerian anomalies in menstruating adolescent girls: a report of 22 cases. J Pediatr Adolesc Gynecol 31:252-257

2. Molina ER, Navas Martinez MC, Castillo OA (2014) Vaginal Gartner cysts: clinical report of four cases and a bibliographic review. Arch Esp Urol 67:181-184

3. Burdan F, Dworzanski W, Cendrowska-Pinkosz M, Burdan M, Dworzanska A (2016) Anatomical eponyms-unloved names in medical terminology. Folia Morphol (Warsz) 75:413-438

4. Inocencio G, Azevedo S, Braga A, Carinhas MJ (2013) Large Gartner cyst. BMJ Case Rep. https:// doi.org/10.1136/bcr-2012-007996

5. Vlahovic A, Stankovic ZB, Djuricic S, Savic D (2014) Giant Gartner duct cyst and elevated CA-125. JPediatr Adolesc Gynecol 27:e137-e138

6. Bats AS, Metzger U, Le Frere-Belda MA, Brisa $M$, Lecuru $F$ (2009) Malignant transformation of Gartner cyst. Int J Gynecol Cancer 19:1655-1657
7. Dzikonski M, Raber G, Kohly A (1979) Gartner Gang - Karzinom der Scheide in der Schwangerschaft. Zentralbl Gynäkol 101:1595-1599

8. Baker KS, Barish M (2014) Inadvertent intravesicular placement of a vaginal contraceptive ring: a case report and review of literature. J Radiol Case Rep 8:22-28 